Trauma Berufskrankh 2010 · 12 [Suppl 1]:70-74 DOI 10.1007/s10039-009-1505-2

Online publiziert: 24. Juni 2009

๑) Springer Medizin Verlag 2009

\author{
W. Hasse ${ }^{1} \cdot$ R. Flieger ${ }^{2} \cdot$ H.-R. Kortmann ${ }^{3}$ \\ ${ }^{1}$ Sektion Traumatologische Intensivmedizin, Klinik für Unfallchirurgie \\ und Orthopädie, BG-Unfallklinik Duisburg GmbH \\ ${ }^{2}$ Sektion Rückenmarkverletzungen, Klinik für Unfallchirurgie \\ und Orthopädie, BG-Unfallklinik Duisburg GmbH \\ ${ }^{3}$ Klinik für Unfallchirurgie und Orthopädie, BG-Unfallklinik Duisburg GmbH
}

\title{
Wenn die „Pumpe“ versagt...
}

\section{Akuter intrathekaler Baclofenentzug - eine lebensbedrohliche Situation}

Die intrathekale Baclofentherapie gewinnt in der Therapie der schweren Spastik eine zunehmende Bedeutung. Sie führt gegenüber der oralen Baclofenmedikation zu einer effektiveren Kontrolle pathologischer Reflexmechanismen bei geringeren $\mathrm{Ne}$ benwirkungen.

Es darf aber nicht übersehen werden, dass dem Verfahren spezifische Komplikationsmöglichkeiten innewohnen, die unerkannt zu erheblicher Morbidität und Letalität führen können. Dies gilt v. a. für das akute Baclofenentzugssyndrom. Kenntnisse über die mit der intrathekalen Baclofentherapie verbundenen Risiken werden mit zunehmender Verbreitung des Verfahrens für den Notfall- und Intensivmediziner immer wichtiger.

Im vorliegenden Beitrag wird deshalb über den Verlauf des akuten intrathekalen Baclofenentzugssyndroms bei 2 tetraplegischen Patienten berichtet.

\section{Kasuistik}

\section{Fall 1}

Ein 55-jähriger männlicher Patient erlitt durch Treppensturz eine $\mathrm{HWK}_{3} / 4$ Berstungsfraktur (HWK: Halswirbelkörper) mit kompletter spastischer Tetraplegie. Nach mehrmonatiger Erstrehabilitation wurde er in die häusliche Pflege entlassen. Im Verlauf kam es zur Verschlim- merung der Spastik, die schließlich eine Mobilisation und Pflege durch die Ehefrau unmöglich machte. Da Erhöhungen der oralen Baclofenmedikation wegen Ateminsuffizienz, Hypotonie und Somnolenz nicht toleriert wurden, wurde eine programmierbare Baclofenpumpe implantiert - mit zufrieden stellender Spastikkontrolle unter Zufuhr von $100 \mu \mathrm{g} \mathrm{Ba-}$ clofen/Tag.

Nach weiteren 5 Monaten musste der Patient wegen eines Infektes der Implantattasche erneut stationär aufgenommen werden. Zur Verhinderung einer aufsteigenden Infektion wurden Pumpe und Katheter explantiert. Prophylaktisch wurde eine spasmolytische Therapie mit Tizanidin begonnen, der unauffällige, fieberfreie Patient auf einer Normalstation überwacht. Am Folgetag klagte er jedoch zunehmend über Unruhe und Atemnot, er musste auf die Intensivstation übernommen werden. Hier zeigten sich $24 \mathrm{~h}$ nach Explantation der Baclofenpumpe spontane Kloni und extreme ReboundSpastiken mit Einschränkung der Ventilation. Es fand sich ein rascher Temperaturanstieg auf $38,8^{\circ} \mathrm{C}$, begleitet von supraventrikulären Tachykardien bis 220/ min und einer Kreislaufdysregulation mit hyper- und hypotensiven Phasen. Trotz mehrfacher i.v. Gabe von 5 mg Diazepam konnte keine Besserung der Spastik oder der vegetativen Entgleisung erzielt wer- den. Es wurde deshalb mit der kontinuierlichen Zufuhr von niedrig dosiertem Propofol begonnen. Darunter besserte sich die vegetative Symptomatik sofort, der Patient blieb jederzeit weckbar. Die Spastik konnte soweit beherrscht werden, dass von kontrollierter Beatmung auf ein assistierendes Verfahren umgestellt werden konnte, die zur Therapiekontrolle durchgeführten CK- (Kreatinkinase-) und Myoglobinbestimmungen zeigten Normalwerte. Zeitgleich wurde mit hochdosierter enteraler Baclofensubstitution (4-mal $20 \mathrm{mg} / \mathrm{Tag}$ ) begonnen, diese musste im Verlauf aber wegen zunehmender Müdigkeit des Patienten auf 4-mal $10 \mathrm{mg} /$ Tag reduziert werden. Hierunter konnte der $\mathrm{Pa}$ tient vom Respirator entwöhnt werden.

Die vom Patienten gewünschte Reimplantation der Baclofenpumpe ist nach Infektsanierung zwischenzeitlich erfolgt.

\section{Fall 2}

Ein 36-jähriger männlicher Patient erlitt durch HWK4/5-Fraktur eine inkomplette Querschnittlähmung unterhalb $\mathrm{C}_{4}$ (Halswirbel 4) mit schmerzhafter beinbetonter Spastik. Bei unzureichender Wirkung oraler Spasmolytika erfolgte die Implantation einer gasdruckbetriebenen Medikamentenpumpe zur intrathekalen Baclofentherapie. Der Patient konnte dadurch rollstuhlfähig in die ambulante Betreu- 
ung entlassen werden. Die Kontrolltermine der intrathekalen Baclofentherapie wurden in einer allgemeinärztlichen Praxis wahrgenommen. Noch innerhalb des letzten Füllungsintervalls musste der $\mathrm{Pa}$ tient 2-mal notärztlich behandelt werden. Es fand sich beide Male das Bild einer autonomen Dysreflexie mit therapierefraktärer Hypertension bei übervoller Blase. Auslöser waren jeweils gescheiterte Versuche der Blasenkatheterisierung durch den Patienten selbst. Da es auch dem Notfallteam aufgrund extremer bein- und beckenbetonter Spastik beide Male nicht gelang, die Blase des Patienten zu entlasten, wurde bei Blutdruckwerten bis $240 \mathrm{~mm}$ $\mathrm{Hg}$ systolisch die stationäre Aufnahme erforderlich. Die urologische Intervention führte jeweils zur raschen Normalisierung der Kreislaufverhältnisse, der Patient konnte nach der ersten Episode sofort entlassen werden. Beim zweiten Ereignis blieb trotz Katheterisierung und Ausschluss eines Harnwegsinfektes eine auffällige vegetative Symptomatik bestehen. Der Patient klagte über schmerzhafte Spastik und quälenden Pruritus, den er als verbrennend charakterisierte. Begleitet wurde die Symptomatik von einem Temperaturanstieg auf $39,7^{\circ} \mathrm{C}$. Eine auf Volumentherapie refraktäre Sinustachykardie (172/min) mit Kreislaufversagen, Atemnot und Hypoxämie machte die Intubation und Beatmung des Patienten erforderlich. Unter Propofolsedierung sistierte die Spastik, das Fieber war rasch rückläufig, die Lungenfunktion besserte sich. Bei anhaltendem Vasopressorund Hydrokortisonbedarf erfolgten unter der Verdachtsdiagnose eines septischen Schocks eine kalkulierte Antibiotikatherapie und intensive Sepsisdiagnostik, jedoch ohne Fokusnachweis. Abstriche und Blutkulturen blieben steril. In der Labordiagnostik fanden sich dagegen hohe CK(1025 U/l) und Myoglobinkonzentrationen $(2388 \mathrm{ng} / \mathrm{ml})$.

Als mögliche Auslöser der Rhabdomyolyse wurden ein intrathekaler Baclofenentzug oder ein Propofolinfusionssyndrom erwogen. Mit Beendigung der Propofolsedierung entwickelten sich eine schwere Rebound-Symptomatik mit Temperaturanstieg auf über $41^{\circ} \mathrm{C}$, ein Linksschenkelblock im EKG (Elektrokardiogramm) sowie ein tachykardes, katechol-
Tab. 1 Ursachen des akuten intrathekalen Baclofenentzugs

\begin{tabular}{|c|c|}
\hline \multirow[t]{4}{*}{ Pumpe } & Batterieausfall \\
\hline & Leerlaufen \\
\hline & Unkontrollierte Förderrate \\
\hline & Stillstand \\
\hline \multirow[t]{6}{*}{ Katheter } & Dislokation \\
\hline & Leckage \\
\hline & Abknickung \\
\hline & Torquierung \\
\hline & Obstruktion \\
\hline & Granulationsbildung \\
\hline \multirow[t]{6}{*}{ Anwender } & Alarmdeaktivierung \\
\hline & Zu niedrige Alarmgrenze \\
\hline & Dosierungsfehler \\
\hline & Programmierfehler \\
\hline & Konzentrationswechsel \\
\hline & Versäumen der Fülltermine \\
\hline
\end{tabular}

Tab. 2 Symptome des akuten intrathekalen Baclofenentzugs

Starke Zunahme der Spastik

(Rebound-Spastik)

Motorisch hyperaktiver Zustand

Parästhesien

Pruritus

Starke Kopfschmerzen

Vegetative Entgleisung mit Schwitzen,

Blasenentleerungsstörung, Tachykardie

Hypertension

Hypotension

Schock

Linksschenkelblock

Kardiomyopathie

Ateminsuffizienz

Stridor

Agitiertheit

Intermittierender Bewusstseinsverlust

Krampfanfälle

Fieberanstieg bis über $40^{\circ} \mathrm{C}$

Rhabdomyolyse mit akutem Nierenversagen

Verbrauchskoagulopathie

Multiples Organversagen

Exitus

aminrefraktäres Herz-Kreislauf-Versagen. Der Patient verstarb unter Reanimation, bevor eine Abklärung der Differenzialdiagnosen möglich war.

\section{Diskussion}

Ein erheblicher Anteil querschnittgelähmter Patienten entwickelt eine schwere, häufig schmerzhafte Spastik mit Einschränkungen der Mobilität und erhöhtem Ri-
Trauma Berufskrankh 2010 - 12 [Suppl 1]:

70-74

DOI 10.1007/s10039-009-1505-2

(C) Springer Medizin Verlag 2009

W. Hasse - R. Flieger · H.-R. Kortmann

Wenn die „Pumpe“ versagt.... Akuter intrathekaler Baclofenentzug - eine lebensbedrohliche Situation

\section{Zusammenfassung}

Die intrathekale Baclofentherapie hat einen unschätzbaren Stellenwert in der Behandlung der Spastik nach traumatischer Querschnittlähmung. Trotz ihres breiten Einsatzes ist die Kenntnis über die damit verbundenen spezifischen und möglicherweise lebensbedrohlichen Komplikationen im Bereich der Intensivmedizin noch sehr unterentwickelt. Wir berichten daher über 2 tetraplegische Patienten mit akutem intrathekalem Baclofenentzugssyndrom und diskutieren anhand der Literatur und eigener Erfahrungen die Symptomatik und Behandlung dieser lebensbedrohlichen Situation.

\section{Schlüsselwörter}

Traumatische Querschnittlähmung · Spastik . Intrathekale Baclofentherapie · Akutes intrathekales Baclofenentzugssyndrom · Therapeutische Maßnahmen

\section{Pump failure.... Acute intra- thecal baclofen withdrawal - a life-threatening event}

\section{Abstract}

Intrathecal baclofen therapy has become an integral part of the treatment of spasticity in traumatic paraplegia. Despite its widespread use there is still a lack of knowledge regarding the associated specific and possibly lifethreatening complications in the field of intensive medicine. We report on the cases of 2 tetraplegic patients with acute intrathecal baclofen withdrawal syndrome and discuss the symptoms and treatment of this life-threatening event on the basis of the literature and own experience.

\section{Keywords}

Spinal cord injury · Spasticity · Intrathecal baclofen therapy - Acute intrathecal baclofen withdrawal syndrome $\cdot$ Therapeutic measures 
Notfallmaßnahmen bei Baclofen-Unterdosierung/Entzug

Lebenserhaltende Maßnahmen einleiten, falls diese indiziert sind.

$\downarrow$

An Patienten, die mit Baclofen-Injektionen behandelt werden und bei denen Zeichen und Symptome eines Baclofen-Entzugs auftreten (Exaggerierte Rebound-Spastik und Muskelstarre, Rhabdomyolyse und Multiorganversagen), soliten die nachfolgend beschriebenen Maßnahmen durchgeführt werden. Diese Maßnahmen entsprechen den Empfehlungen einer Kommission aus in dieser Therapie erfahrenen Ärzten, die zur Erforschung dieser Thematik gebildet wurde: ${ }^{1,2}$

1. Sofort einen mit Baclofen-Injektionen erfahrenen Arzt konsultieren, vorzugsweise den behandelnden Arzt des betreffenden Patienten, den Empfehlungen dieses Arztes folgen. Dieser Schritt ist auch bei scheinbar leichten Zeichen und Symptomen wichtig.

2. Ist kein mit Baclofen-Injektionen erfahrener Arzt erreichbar, solite mindestens eine der folgenden Möglichkeiten in Betracht gezogen werden, sofern keine Kontraindikation vorliegt:

- orale* oder enterale Baclofengabe in hoher Dosierung

- Wiederherstellung der intrathekalen Baclofeninfusion

- intravenöse Verabreichung von Benzodiazepinen durch fortlaufende oder

intermittierende Infusion unter Titration der Dosierung, bis sich die gewünschte therapeutische Wirkung einstellt.

* Hinweis: de orale Baclofengabe sollte nicht als einzige Methode zur Behandlung eines Entzugssyndroms in Verbindung mit Baclofeninjektion angewandt werden.

$\downarrow$

Melden Sie den Vorfall bei Medtronic.

Abb. $1 \Delta$ Auszug der Synchromedgebrauchsanweisung (mit freundlicher Genehmigung der Fa. Medtronic)

siko für Kontrakturen, Verletzungen oder Dekubitalulzera. Fast alle Patienten benötigen neben der Physiotherapie eine medikamentöse Behandlung. Wenn orale Spasmolytika trotz maximaler Dosierung nicht ausreichen oder nicht gut toleriert werden, bietet die intrathekale Baclofentherapie eine hervorragende Alternative [15]. Das Verfahren findet deshalb zunehmend Anwendung, Kenntnisse über Komplikationsmöglichkeiten sind dagegen nicht sehr weit verbreitet [27]. Dies birgt v. a. dann Risiken, wenn ein Patient im Notfall außerhalb der ihn betreuenden Einrichtung behandelt werden muss. Dabei scheint die akzidentelle intrathekale Baclofenüberdosierung mit Somnolenz, Hypotension und Hyporeflexie dem Intensivmediziner nur geringe Probleme zu bereiten. Sie tritt typischerweise nach Dosissteigerung auf und verläuft unter symptomatischer Behandlung selbstlimitierend. Anders dagegen verhält es sich mit dem intrathekalen Baclofenentzugssyndrom, das unerkannt oder unzureichend behandelt mit hoher Letalität einhergeht.

\section{Ursachen des intrathekalen Baclofenentzugs}

Bereits mit Einführung der peroralen Therapie wurde über Baclofenentzugssyn- drome nach geplanter oder ungeplanter Unterbrechung der Medikation berichtet $[9,12,19,22]$. Das Erkennen eines intrathekalen Baclofenentzugs scheint ungleich schwieriger zu sein [25].

Nach Publikation letaler Verläufe [ 7 , 13] wurden alle verfügbaren Berichte über intrathekale Baclofenentzugssyndrome durch Coffey et al. [2] ausgewertet. Mit 6 Todesfällen in 16 Kasuistiken fand sich eine hohe Letalität [2]. Die Autoren vermuteten, dass v. a. Patienten mit Querschnittlähmung oberhalb von Th6 (Brustwirbel 6) und autonomer Dysreflexie ein hohes Risiko für die Entwicklung des lebensbedrohlichen Baclofenentzugssyndroms haben. Die Ergebnisse führten zu einem Safety Alert der amerikanischen Food and Drug Administration (FDA) im Jahr 2002 (Infobox 1). Der Warnhinweis wurde in die Produktinformationen der Hersteller aufgenommen, dennoch wurden und werden weiterhin Fälle berichtet, eine relevante Dunkelziffer ist wahrscheinlich. Die Ursache dafür kann in der Komplexität des Systems aus Pumpe, Katheter, Patient und Behandler liegen. Von der Einstellung der Alarmgrenzen [18] bis hin zur perioperativen Migration des Katheters [28] sind vielfältige Fehlermöglichkeiten gegeben (• Tab. 1).
Wegen dieser kaum überschaubaren Risiken wurden Protokolle zur standardisierten funktionellen und radiologischen Überprüfung vorgeschlagen [20]. Aber auch diese konnten in Einzelfällen eine Fehlfunktion weder sicher detektieren noch ausschließen [24]. Im Zweifelsfall ist daher auch bei unauffälliger Funktionsprüfung eine operative Exploration erforderlich.

\section{Pathophysiologie und Symptome des akuten intrathekalen Baclofenentzugs}

Baclofen ist ein Derivat der $\gamma$-Aminobuttersäure (GABA) mit spezifischer Affinität zu den $\mathrm{GABA}_{\mathrm{B}}$-Rezeptoren im Hinternhorn des Rückenmarkes, im Hirnstamm und in Arealen des Kleinhirns. Obwohl die molekularen und zellulären Mechanismen des Baclofenentzugs bisher nicht gut untersucht sind, wird angenommen, dass nach längerer Baclofentherapie der akute Entzug zur überschießenden Enthemmung aller $\mathrm{GABA}_{\mathrm{B}}$-inhibierten katecholaminergen Neurotransmittersysteme führt $[11,14]$.

Die Symptome des intrathekalen Baclofenentzugs (• Tab. 2) umfassen daher einen weiten Bereich vegetativer, zentralnervöser, neuromuskulärer und kardiovaskulärer Entgleisungen bis hin zur Kardiomyopathie [16]. In leichteren Verläufen mit Pruritus und Rückkehr zur vorbestehenden Spastizität ähnelt die Symptomatik einer Toleranzentwicklung [26]. Das schwerere lebensbedrohliche Entzugssyndrom gleicht dagegen nach Auffassung einiger Autoren in seiner Symptomatik dem Malignen Neuroleptischen Syndrom oder dem Serotoninsyndrom $[11,14,21]$.

\section{Fallstricke in der Differenzialdiagnostik}

Unsere Erfahrung bestätigt Berichte anderer Untersucher, dass der schwere Baclofenentzug mit Fieber, Tachykardie, Kreislaufinstabilität und deliranter Symptomatik häufig als Allgemeininfektion oder Sepsis fehlgedeutet wird [10, 17, 19]. Da beim Querschnittgelähmten die Vermutung nahe liegt, dass die Spastik durch eine Infektion verstärkt wurde, kann es leicht zur Verwechslung von Ursache und 
Wirkung kommen [4, 15]. Mitverantwortlich dafür ist, dass die vegetativen Entgleisungen typischerweise erst 1-3 Tage nach Unterbrechung der Baclofenzufuhr auftreten.

Obwohl bei unserem ersten Patienten die abrupte Beendigung der intrathekalen Baclofenzufuhr offensichtlich war, bewirkte der fieberhafte Verlauf dennoch eine Verunsicherung der Behandelnden. Im zweiten Fall waren die septischen Symptome Fieber, Tachykardie und Kreislaufinstabilität so dominierend, dass neben der intensiven Fokussuche die Abklärung der Differenzialdiagnose in Verzug geriet. Auch in den Fällen von Coffey et al. [2] führte diese Verkennung zu einem fatalen Ausgang. Da selbst subtile radiologische und funktionelle Überprüfungen eine Fehlfunktion der Baclofenpumpe nicht mit Sicherheit ausschließen können, muss wegen der imminenten Lebensbedrohung letztlich auf anderem Wege eine Abklärung erzwungen werden, z. B. durch probatorische intrathekale Baclofeninjektion oder operative Revision des Systems.

\section{Symptomatische und kausale Therapie}

Bei Unterbrechung einer länger bestehenden intrathekalen Baclofenzufuhr wird zur Verhinderung eines Entzugssyndroms eine hohe perorale Substitution mit Baclofen kombiniert mit Benzodiazepinen empfohlen. Gute Ergebnisse wurden auch mit dem Serotoninantagonisten Cyproheptadin [14] berichtet. Bei unzureichender Symptomkontrolle kann unter intensivmedizinischer Behandlung durch niedrig dosiertes Propofol eine Kupierung der vegetativen und neuromuskulären Entgleisung erreicht werden [1].

Die von Coffey et al. [2] publizierten Fälle zeigen aber, dass die symptomatische Therapie allein nicht ausreicht, um beim schweren intrathekalen Baclofenentzugssyndrom letale Verläufe sicher zu verhindern. Höchste Priorität hat daher die Überprüfung des Gesamtsystems mit rascher Wiederherstellung einer intrathekalen Baclofenzufuhr [14]. Es wird deshalb in den Produktinformationen der Herstel- ler empfohlen, sofort einen erfahreneren Behandler hinzuziehen (• Abb. 1).

Für die rasche kausale Therapie bieten sich die Bolusfunktion der batteriebetriebenen Pumpen oder der Sideport der gasdruckbetriebenen Systeme an, einen intakten und korrekt liegenden Katheter vorausgesetzt. Bleibt der Erfolg aus, muss auch bei sonst unauffälliger Pumpe von einer Fehlfunktion ausgegangen werden, und es sollte eine chirurgische Exploration erfolgen. Überbrückend kann durch intrathekale Gabe von 50-100 $\mu \mathrm{g}$ Baclofen, alternativ als Single-Shot [23] oder über Spinalkatheter [6], eine lebensrettende Symptomkontrolle erzielt werden. Die nachfolgende Besserung der Symptomatik kann als beweisend für eine Fehlfunktion gelten.

Besondere Schwierigkeit bereitet das Vorgehen bei infizierten Kathetersystemen, da die orale Substitution eine Entzugssymptomatik nicht sicher verhindert [8]. Einige Autoren empfehlen die Fortsetzung der intrathekalen Baclofenzufuhr unter systemischer und intrathekaler Antibiotikagabe [5, 6] oder die überlappende perorale Einstellung mit Ausschleichen der intrathekalen Baclofenzufuhr („tapering“). Eine mögliche Therapiekontrolle ergibt sich neben der Überprüfung der Vitalparameter durch regelmäßige Serumkontrollen von CK und Myoglobin [3].

\section{Vigilanz und Prophylaxe}

Coffey et al. [2] stellten fest, dass milde Formen des Baclofenentzugs mit Rückkehr zur vorbestehenden Spastizität leichter erkannt und schneller behandelt wurden als das schwere lebensbedrohliche Entzugssyndrom. Sie forderten daher eine verbesserte Information und Schulung von Patienten, Betreuern, Angehörigen und Behandlern. Die Risikogruppe hoch querschnittgelähmter Patienten sollte zusätzlich mit einer oralen Notfallmedikation ausgestattet sein und einen Notfallausweis mit sich führen, auf dem Erstmaßnahmen und Notfalltelefonnummern vermerkt sind.

\section{Fazit}

Der akute intrathekale Baclofenentzug stellt eine Notfallsituation dar, die bei Verkennung oder inadäquater Behandlung mit hoher Letalität verbunden ist. Die Koinzidenz von zunehmender Spastik, vegetativer Entgleisung mit Tachykardie, Hyper- oder Hypotension, Fieber und Rhabdomyolyse muss bei Trägern von Baclofenpumpen immer an die Möglichkeit eines intrathekalen Baclofenentzugs denken lassen. Es muss die Hilfe eines mit dieser Komplikation vertrauten Arztes gesucht und bereits bei Verdacht eine enterale Substitution mit hochdosiertem Baclofen begonnen werden. Unter intensivmedizinischer Überwachung werden bei progredienter Symptomatik adjuvant Benzodiazepine oder auch Propofol eingesetzt. Zur Beurteilung des Schweregrades und des Therapieerfolges haben sich die Laborparameter Myoglobin oder CK bewährt. Die rasche Wiederherstellung der intrathekalen Baclofenzufuhr gilt als einzig kausale Maßnahme beim foudroyant verlaufenden Baclofenentzugssyndrom.

\section{Infobox 1 Weblinks}

www.fda.gov/medwatch/safety/2002/baclofen.htm

\section{Korrespondenzadresse}

\section{Dr.W. Hasse}

Sektion Traumatologische Intensivmedizin, Klinik für Unfallchirurgie und Orthopädie, BG-Unfallklinik Duisburg GmbH, Großenbaumer Allee 250, 47249 Duisburg werner.hasse@bgu-duisburg.de

Interessenkonflikt. Der korrespondierende Autor gibt an, dass kein Interessenkonflikt besteht.

\section{Literatur}

1. Ackland GL, Fox R (2005) Low-dose propofol infusion for controlling acute hyperspasticity after withdrawal of intrathecal baclofen therapy. Anesthesiology 103:663-665

2. Coffey RJ, Edgar TS, Francisco GE et al (2002) Abrupt withdrawal from intrathecal baclofen: recognition and management of a potentially life-threatening syndrome. Arch Phys Med Rehabil 83:735741

3. Colachis SC, Rea GL (2003) Monitoring of creatinine kinase during weaning of intrathecal baclofen and with symptoms of early withdrawal. Am J Phys Med Rehabil 82:489-492 
4. Cunningham JA, Jelic S (2005) Baclofen withdrawal: a cause of prolonged fever in the intensive care unit. Anaesth Intensive Care 33:534-537

5. Douglas AF, Weiner HL, Schwartz DR (2005) Prolonged intrathecal baclofen withdrawal syndrome. J Neurosurg 102:1133-1136

6. Duhon BS, MacDonald JD (2007) Infusion of intrathecal baclofen for acute withdrawal. Technical note. J Neurosurg 107:878-880

7. Green LB, Nelson VS (1999) Death after acute withdrawal of intrathecal baclofen: case report and literature review. Arch Phys Med Rehabil 80:16001604

8. Greenberg MI, Hendrickson RG (2003) Baclofen withdrawal following removal of an intrathecal baclofen pump despite oral baclofen replacement J Toxicol Clin Toxicol 41:83-85

9. Harrison SA, Wood CA (1985) Hallucinations after preoperative baclofen discontinuation in spinal cord injury patients. Drug Intell Clin Pharm 19:747-749

10. Kao LW, Amin Y, Kirk MA, Turner MS (2003) Intrathecal baclofen withdrawal mimicking sepsis. J Emerg Med 24:423-427

11. Leo RJ, Baer D (2005) Delirium associated with baclofen withdrawal: a review of common presentations and management strategies. Psychosomatics 46:503-507

12. Mandac BR, Hurvitz EA, Nelson VS (1993) Hyperthermia associated with baclofen withdrawal and increased spasticity. Arch Phys Med Rehabil 74:9697

13. Meinck HM, Tronnier V, Rieke K et al (1994) Intrathecal baclofen treatment for stiff-man syndrome: pump failure may be fatal. Neurology 44:22092210

14. Meythaler JM, Roper JF, Brunner RC (2003) Cyproheptadine for intrathecal baclofen withdrawal. Arch Phys Med Rehabil 84:638-642

15. Ochs G (2004) Komplikationen, Nebenwirkungen und Risiken. In: Ochs G (Hrsg) Die Behandlung der schweren Spastizität. Baclofen intrathekal - Botulinumtoxin. Leitfaden für die praktische Anwendung. Thieme, Stuttgart, S 84-96

16. Pizon AF, LoVecchio F (2007) Reversible cardiomyopathy complicating intrathecal baclofen withdrawal: a case report. J Med Toxicol 3:187-189

17. Reeves RK, Stolp-Smith KA, Christopherson MW (1998) Hyperthermia, rhabdomyolysis and disseminated intravascular coagulation associated with baclofen pump catheter failure. Arch Phys Med Rehabil 79:353-356

18. Rigoli G, Terrini G, Cordioli Z (2004) Intrathecal baclofen withdrawal syndrome caused by low residual volume in the pump reservoir: a report of 2 cases. Arch Phys Med Rehabil 85:2064-2066

19. Rivas DA, Chancellor MB, Hill K, Freedman MK (1993) Neurological manifestations of baclofen withdrawal. J Urol 150:1903-1905

20. Roche N, Even-Schneider A, Bussel B, Bensmail D (2007) Conduite à tenir devant une recrudescence de spasticité chez un patient porteur d'une pompe à baclofène. Ann Readapt Med Phys 50:93-99

21. Salazar ML, Eiland LS (2008) Intrathecal baclofen withdrawal resembling serotonin syndrome in an adolescent boy with cerebral palsy. Pediatr Emerg Care 24:691-693

22. Sampathkumar P, Scanlon PD, Plevak DJ (1998) Baclofen withdrawal presenting as multiorgan system failure. Anesth Analg 87:562-563

23. Samson-Fang L, Gooch J, Norlin CH (2000) Intrathecal baclofen withdrawal simulating neuroleptic malignant syndrome in a child with cerebral palsy. Dev Med Child Neurol 42:561-565
24. Schurch B (1993) Errors and limitations of the multimodality checking methods of defective spinal intrathecal pump systems. Case report. Paraplegia 31:611-615

25. Siegfried RN, Jacobson L, Chabal C (1992) Development of an acute withdrawal syndrome following the cessation of intrathecal baclofen in a patient with spasticity. Anesthesiology 77:1048-1050

26. Smail DB, Hugeron C, Denys P, Bussel B (2005) Pruritus after intrathecal baclofen withdrawal: a retrospective study. Arch Phys Med Rehabil 86:494497

27. Tronnier VM, Bardutzky J (2008) Intrathekale Therapie, Pumpen, Pumpenversagen. In: Schwab S, Schellinger P, Werner C et al (Hrsg) Neurolntensiv. Springer, Heidelberg, S 371-378

28. Vaidyanathan S, Soni BM, Oo T et al (2003) Bladder stones - red herring for resurgence of spasticity in a spinal cord injury patient with implantation of Medtronic Synchromed pump for intrathecal delivery of baclofen - a case report. BMC Urol 3:3

29. Zuckerbraun NS, Ferson SS, Albright AL, Vogeley E (2004) Intrathecal baclofen withdrawal: emergent recognition and management. Pediatr Emerg Care 20:759-764 